\title{
Visual Segmentation and Localization of Mobile Robots Based on the Feedback Regulation
}

\author{
Rong Zhao ${ }^{1}$, Mingxin Yuan ${ }^{1,2, *}(\varpi)$, Xiaobin Hua ${ }^{1}$, Yi Shen ${ }^{1,2}$, Peng $\mathrm{Li}^{1}$ \\ ${ }^{1}$ (School of Mechanics and Automotive Engineering,Jiangsu University of Science and Technology, \\ Zhangjiagang, China) \\ ${ }_{2}^{2}$ (Suzhou Institute of Technology, Jiangsu University of Science and Technology, Zhangjiagang, China)
}

\begin{abstract}
In order to realize the rapid and accurate localization of multiple mobile robots under the global vision, a new visual segmentation algorithm based on the feedback adjustment of threshold is presented in paper. First, the position information of the mobile robots is described by the color label plates which are designed by ourselves; then, the improved grid method is used to search the color labels; finally, the segmentation threshold of the next frame image is adjusted based on the segmentation results of the mobile robots and the amount of noise in the current frame, and the visual localization of multiple mobile robots is realized through the self-adjusting thresh segmentation of color label plates. The experiment results show that the proposed method is characterized by small calculation amount, good real-time and strong noise suppression capability, performance and has strong noise suppression abilities, and the accurate visual localization of multiple mobile robots under the global vision can be realized.
\end{abstract}

Keywords: Feedback adjustment; Threshold segmentation; Grid method; Visual localization

\section{Introduction}

Localization is a key prerequisite in the autonomous navigation of mobile robots. In order to obtain more accurate localization information of the mobile robots, the visual localization based on the camera sensors becomes the research focus in the last few years. The visual localization under the global camera has attracted much attention for its small computation amount and accurate localization, especially is used widely in the fields of soccer robots. At present, the visual localization of the soccer robots under the global vision mainly depends on the color label plates on the top of the robots. In order to segment the color label plates, Liu et al. ${ }^{[1]}$ achieved the different threshold value at the different regions of an image through the background subtraction method and the pattern matching method, and the self-adaptive segmentation was realized along with the change of pixel coordinates, which shows good regional self-learning ability; however, the threshold value can not be adjusted along with the change of time. Aiming at the small-size soccer robot system, Zhu et al ${ }^{[2]}$ presented a visual segmentation algorithm based on the neural network. First of all, the threshold value and the weights of the BP network are trained based on the sample images; then, the image processing and the image recognition are realized. The experiment results show that the network segmentation algorithm owns better stability and can reduce the error rate of recognition; however, it is difficulty to achieve the complete sample data.

From the current study, for the mobile robot vision system with high real-time requirement, the threshold method has became the main method in the image segmentation due to its simple model, small computation amount and good stability, and so on. The common methods include: bimodal histogram method ${ }^{[3]}$, OSTU method ${ }^{[4]}$, 2-D maximum entropy image segmentation method ${ }^{[5]}$ and so on. Histogram algorithm is mainly suitable for the images which have clear bimodal characteristics. However, the segmentation effect of the images which have a lot of noise and multipeak histogram will be reduced for the improper threshold value. OSTU method now has became one of the most widely used methods which can adaptively select the segmentation threshold for its strong adaptive ability; however, its calculation amount is very large because all gray pixels need to be processed when achieving the segmentation threshold value; in other words, its real-time performance is not good and it is not suitable for the video image processing. The maximum entropy threshold segmentation method can reduce the disturbance of noise adequately to achieve the maximum information amount of background and targets; however, its calculation amount is still large during obtaining the optimal threshold, and it is also not suitable for the occasions with high real-time performance. To more quickly and accurately achieve the position and angular information of multiple mobile robots under the global vision, on the basis of the color label plate of robots which are designed by ourselves, the segmentation threshold value is adaptively adjusted according to the threshold segmentation results of current flame, which realizes the vision segmentation and localization of multiple mobile robots.

* Correspondence author, mxyuan78@ @otmail.com. 


\section{Definition of the Color Label Plate for the Mobile Robot}

In order to better realize the visual segmentation and localization of multiple mobile robots under the global vision, the color label plates in RoboCup are referenced in paper to express the pose information of the mobile robot. The color label of the mobile robot in this paper is designed based on the round label. The layout of the color label in the color plate is shown in Fig.1. Label 1 is the baseplate, label 2 is the main color label, and label 3 is the auxiliary color label. The coordinate of the main color label 2 is defined as the center coordinate of the mobile robot. The discrimination of different robots mainly depends on the sequence of different colors from the auxiliary color label 3 . The forward direction of the mobile robot is determined by the vector from the main color label to the middle auxiliary color label.

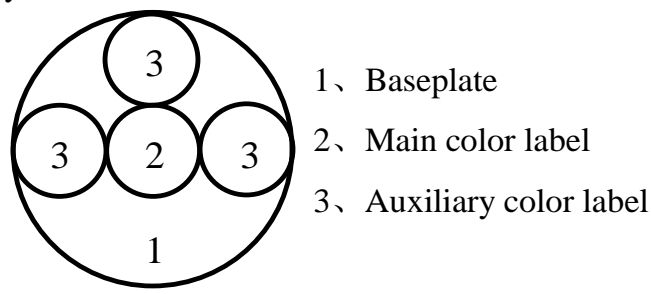

Fig.1. Layout of the robot color label plate

Due to the number of the robots in this system is not more than 6, the main color label is colored with blue and the auxiliary color label is colored with red and yellow. The base plate is colored with black.

\subsection{Image Preprocess of Color Label Plate}

\section{Search Of Color Label Plate Based On Improved Grid Method}

In order to eliminate the interference from the illumination to the segmentation of the color label plate, the RGB color image acquired by image capture card is converted to YUV format firstly according to Eq.(1), and the color of the color label plate is then extracted based on the UV components.

$$
\left[\begin{array}{l}
Y \\
U \\
V
\end{array}\right]=\left[\begin{array}{ccc}
0.299 & 0.587 & 0.114 \\
-0.169 & -0.3316 & 0.500 \\
0.500 & -0.4186 & -0.0813
\end{array}\right]\left[\begin{array}{l}
R \\
G \\
B
\end{array}\right]
$$

To further simplify the data calculation regarding the segmentation of the color label plate, based on the threshold method, we have a four-valued processing on the image from YUV space by using Eq.(2). The value of the background color is set to 0 , the value of the red color label is set to 1 , the value of the yellow color label is set to 2, and the value of the blue color label is set to 3 .

$$
G(x, y)= \begin{cases}1 & \text { if } V_{f(x, y)} \geq V_{\text {red }} \\ 2 & \text { if } U_{f(x, y)} \leq U_{\text {yellow }} \\ 3 & \text { if } U_{f(x, y)} \geq U_{\text {blue }} \\ 0 & \text { else }\end{cases}
$$

Where, $G_{(x, y)}$ is the result after four-valued processing, $V_{\text {red }}$ is the segmentation threshold of the $V$ component for red color, $V_{f(x, y)}$ is the $V$ component value of the pixel $f_{(x, y)}$ in YUV space, $U_{\text {yellow }}$ is the segmentation threshold of the $U$ component for yellow color, $U_{f(x, y)}$ is the $U$ component value of the pixel $f_{(x, y)}$ in YUV space, and $U_{\text {blue }}$ is the segmentation threshold of the $U$ component for blue color.

\subsection{Search of the Color Label Plate}

In order to improve the search efficiency of the color label plate, based on the basic grid method ${ }^{[7]}$, an improved grid search method is put forward according to the features of the designed color label plate. The flow of the improved grid search method is described as follows.

Step 1: Execute the search on the blue color label based on the set horizontal and vertical step length for the grid search.

Step 2: When the value of blue color label is detected, the pixel point is taken as the seed, and the iterative search of 8-nearest neighbor in the connected region is carried out to obtain all the pixels in the region with the same color label value. Then, the sum of the abscissas and the sum of the ordinates of all pixels are calculated, and the area of the single connected region is calculated. When the center coordinate of the connected region is calculated according to the Eqs.(3) and (4), the pixel coordinate of the blue color label is achieved, in other words, we can obtain the center coordinate of the mobile robot. Through the transform from 
the pixel coordinate to the practical space coordinate, we can obtain the coordinate of the mobile robot in the practical space.

$$
\begin{aligned}
X_{\text {robot }} & =\frac{\sum X_{i(x, y)}}{\sum I(x, y)} \\
Y_{\text {robot }} & =\frac{\sum Y_{i(x, y)}}{\sum I(x, y)}
\end{aligned}
$$

Where, $\sum I_{(x, y)}$ is the area of a single connected region. $\sum X_{i(x, y)}$ is the sum of the abscissas of a single connected region and $\sum Y_{i(x, y)}$ is the sum of ordinates of a single connected region.

Step 3: After obtaining the center coordinate $\left(X_{\text {robot }}, Y_{\text {robot }}\right)$ of mobile robot, we take the coordinate as the center of a circle, and have a circular search with the radius $D_{C L}$ which is the diameter of the color label. The circular search is schematically shown in Fig.2.

In the designed color label plate, because the auxiliary color label surrounds the main color label, we just need to search the auxiliary color label in the local area through the circular search, which reduces the time consumption of the search of the auxiliary color label in the whole image, and improves the search efficiency.

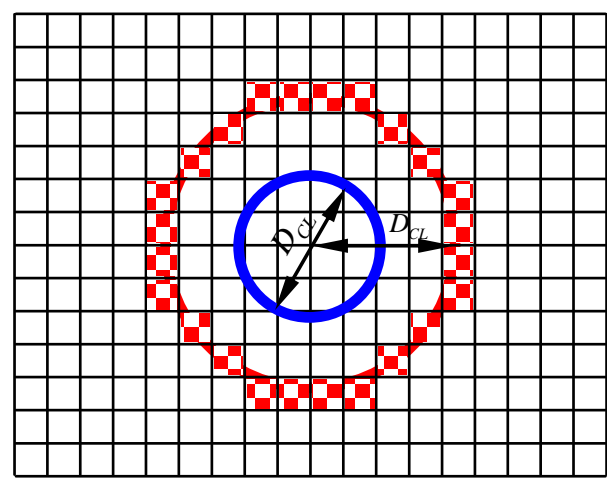

Fig.2. Circular search of auxiliary color label

Step 4: When we search for a pixel regarding the auxiliary color label, the point is taken as the speed, and the iterative search of 8-nearest neighbor in the connected region is carried out again to obtain the center coordinate of the auxiliary color label. After a complete circular search is finished along with the main color label, all coordinates of all auxiliary color labels in the color label plate can be obtained.

Step 5: After obtaining the complete information of a color label plate, the forward direction of a mobile robot can be determinated according to the vector from the main color label to the middle auxiliary color label.

Step 6: Judge whether the searches of all mobile robots are finished. If not, then go to step 1 and continue to complete the search of other robots in the visual area; otherwise exit the search.

\section{Feedback Adjustment of Segmentation Threshold}

In the threshold-based video image segmentation, the selection of the threshold directly determines the classification of pixel points, and it is the key to the image segmentation. In order to improve the accuracy and efficiency of the robot segmentation based on the color label plate, a feedback adjustment algorithm for the segmentation threshold is presented in this paper according to the features of the designed color label plate. Two factors are considered during the adjustment: 1Whether all of the color labels can be segmented through the set threshold value; 2Amount of the noise after the image segmentation under this threshold. The two factors are not independent. When the segmentation threshold is relatively low, the color label which is extracted is more complete; however, the amount of the noise will increase at the same time, which reduces the segmentation precision. On the contrary, when the segmentation threshold is relatively high, the amount of noise will decrease; however, the complete extraction of color label is affected. Therefore, on the promise of a complete segmentation of all the color label plates, the efficiency and accuracy of image segmentation can be increased through the adaptive threshold segmentation.

In conclusion, the basic theory of the feedback regulation of the segmentation threshold can be described as: According to the image segmentation results, (namely, whether all robots are segmented and the amount of the noise), in the current frame, the image segmentation threshold in the next frame can be adjusted. For this purpose, the feedback regulation of segmentation threshold is defined as shown in Eq.(5). 


$$
L_{r t}=L_{b t} \cdot \exp \left(\left(1-\frac{N_{m c l}}{N_{r}}\right)^{\frac{1}{3}}-1\right) \cdot\left(\frac{N_{n} \cdot L_{x} \cdot L_{y}}{N_{m n}}+1\right)
$$

Where, $L_{r t}$ is the actual step length for the threshold adjustment; $L_{b t}$ is the basic step length for the threshold adjustment; $N_{m c l}$ is the number of main color label in actual segmentation; $N_{r}$ is the number of robots which is equal to the number of main color label; $M_{m n}$ is the estimated amount of maximum noise; $M_{n}$ is the amount of noise in actual segmentation; $L_{x}$ and $L_{y}$ are the step length at $x$ direction and y direction in the grid search, respectively.

In this paper, the adjustment step for segmentation threshold is limited in a certain range. If length of the adjustment step is too long, the adjustment of the segmentation threshold likely cause the overshoot and oscillation. On the contrary, if the length of the adjustment step is too short, adjustment speed will be reduced, and the adjustment is not suitable for the video image with rapid changes.

\section{Experiment Result and Analysis}

To verify the validity of the proposed self-adjusting threshold segmentation algorithm, some tests are executed in Visual C++ 6.0 on an Intel Pentium IV $2.99 \mathrm{GHz}$ computer with 2GB RAM. The experiment environment is shown in Fig.3. In the figure, there are two global cameras. On the basis of the two camera and image mosaic technology, we can obtain a wide motion space of mobile robots. In this experiment, to verify the validity of the proposed segmentation algorithm, only one camera is used. What's more, there are some mobile robots with color label plates under the cameras.

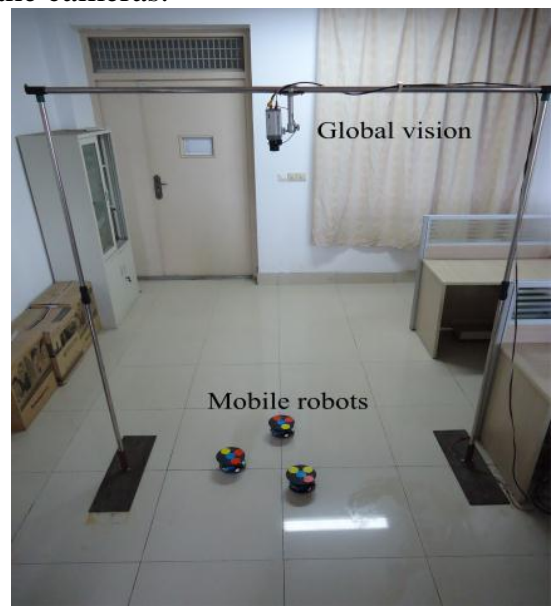

Fig.3. Experiment environment

\subsection{Determination of the Grid Search Step}

From Eq.(5), it can be seen that the self-adjusting visual segmentation mainly depends on two factors: step length of grid search and the amount of noise after segmentation. The former includes the search length at the horizontal direction and vertical direction, and the selection of the length is very crucial to the threshold adjustment. Therefore, aiming at Fig.4, we determine the step length through the amount of noise in this paper. The correspondence between the step length for grid search and amount of noise is shown in table 1.

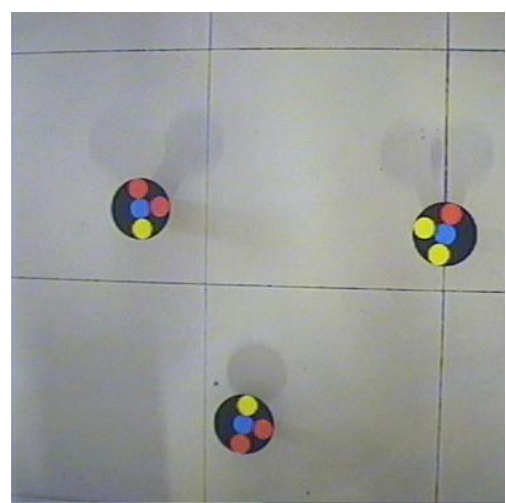

Fig.4. Experiment image for the test of step length 
Table 1. Correspondence between the step length for grid search and amount of noise

\begin{tabular}{|c|c|c|c|c|c|c|c|c|c|c|c|c|}
\hline adjustment step length at Y direction /pixel & 1 & 1 & 1 & 2 & 2 & 2 & 3 & 3 & 3 & 4 & 4 & 4 \\
\hline adjustment step length at X direction /pixel & 1 & 2 & 3 & 1 & 2 & 3 & 1 & 2 & 3 & 1 & 2 & 3 \\
\hline amount of noise which is detected & 38 & 38 & 36 & 16 & 16 & 16 & 13 & 13 & 13 & 7 & 7 & 7 \\
\hline
\end{tabular}

From table1, we can see that the increase of step length at the $\mathrm{x}$ direction has little effect on the amount of noise which is detected; however, the increase at y direction can reduce the amount of noise effectively. When the amount of the noise is small, the effect of reducing the amount noise is not obvious through increasing the step length at any direction; however, if the step length is too long, the target color label will be skipped, which will to the loss of target. Therefore, in paper, the horizontal search step length is 1 and the vertical search step length is 3 .

\subsection{Results of robot segmentation and localization}

After the step length for the grid search is determined, aiming at three different environments as shown in Fig.5(a), Fig.6(a), and Fig.7(a), some localization experiments for the robots based on self-adjust threshold segmentation are executed. The experiment results are shown in Fig.5(b), Fig.6(b) and Fig.7(b). From these figures, it can be seen that all robots are extracted based on the proposed algorithm although there is shadow around the robot, which verifies the validity of the proposed algorithm.

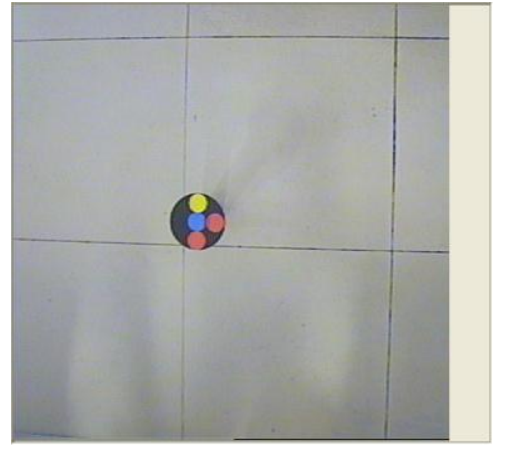

(a) Experiment picture

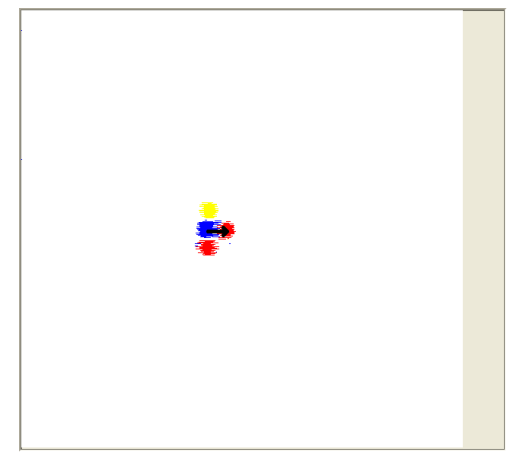

(b) Segmentation result

Fig.5. Experiment result in environment 1

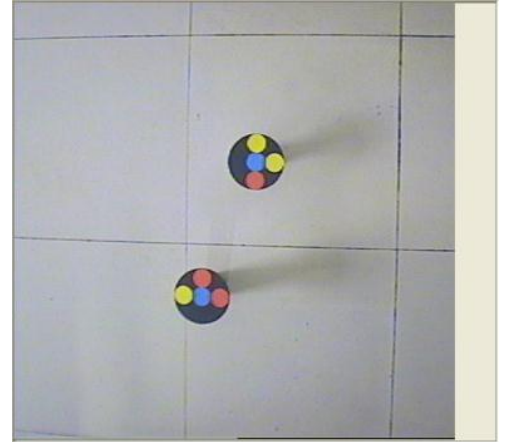

(a) Experiment picture

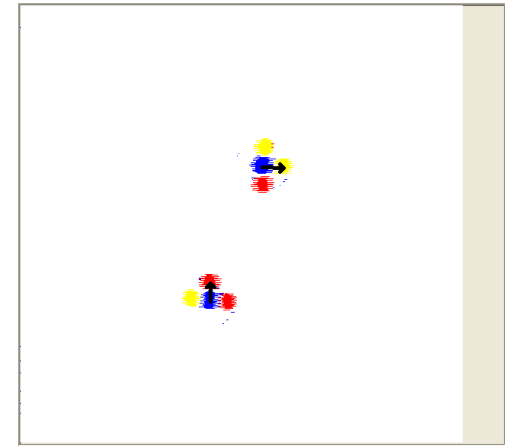

(b) Segmentation result

Fig.6. Experiment result in environment 2

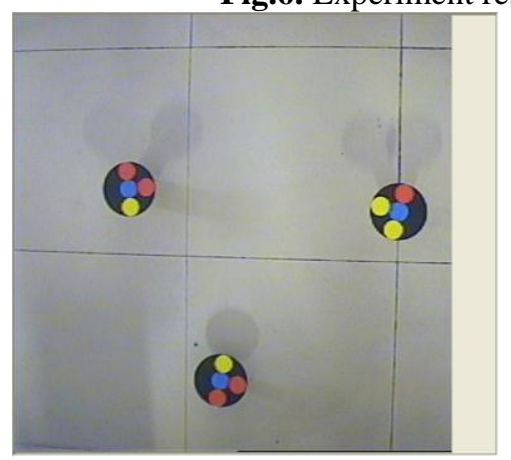

(a) Experiment picture

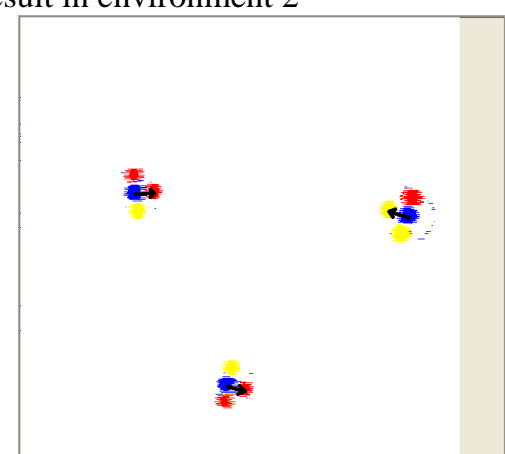

(b) Segmentation result

Fig.7. Experiment result in environment 3 
The localization results of the mobile robot are shown in Table 2, which include the pixel coordinates and direction angle.

Table2. Localization Results in three environments

\begin{tabular}{|c|c|c|c|c|c|c|}
\hline \multirow{2}{*}{$\begin{array}{l}\text { No. of the } \\
\text { robot }\end{array}$} & \multicolumn{2}{|c|}{ Environment 1 } & \multicolumn{2}{c|}{ Environment 2 } & \multicolumn{2}{c|}{ Environment 3 } \\
\cline { 2 - 7 } & $\begin{array}{l}\text { Pixel } \\
\text { coordinate }\end{array}$ & $\begin{array}{l}\text { Direction } \\
\text { angle }\end{array}$ & $\begin{array}{l}\text { Pixel } \\
\text { coordinate }\end{array}$ & $\begin{array}{l}\text { Direction } \\
\text { angle }\end{array}$ & $\begin{array}{l}\text { Pixel } \\
\text { coordinate }\end{array}$ & $\begin{array}{l}\text { Direction } \\
\text { angle }\end{array}$ \\
\hline Robot1 & $/$ & $/$ & $/$ & $/$ & $90,345)$ & 2 \\
\hline Robot2 & $(244,286)$ & 0 & $(248,187)$ & -2 & $(270,92)$ & -14 \\
\hline Robot3 & $/$ & $/$ & $(316,364)$ & $(508,314)$ & 162 \\
\hline
\end{tabular}

\section{Conclusion}

The localization of multiple mobile robots under the global vision has been the research hotspot and difficulty in the field of robotics. In order to further improve the visual segmentation effect of the mobile robots under global vision, a color label plate for the pose information of a mobile robot is designed firstly in this paper. The plate consists of main color label and auxiliary color label. The main color label is surrounded by three auxiliary color labels. Then, an improved method for the grid search is used to improve the search efficiency of color label plate. In the visual segmentation of mobile robots, the adjustment step length for threshold value is defined based on the segmentation results and the amount of noise to realize the multi-robot online adaptive threshold segmentation. Many experiment results in different conditions verify the validity of the proposed self-adjusting threshold segmentation algorithm.

\section{Acknowledgements}

The work was supported by the National Nature Science Foundation of China (NSFC) (No. 61105071), Qing Lan Project of Jiangsu Province, and 2013 Undergraduate Scientific and Technological Innovation Project of Jiangsu Province and China.

\section{References}

[1]. J.L. Liu, Construction of RoboCup Small Size Soccer Robot Vision System, master's diss., Zhejiang University, Hangzhou, China, 2007.

[2]. Y.L. Zhu and X.M. Li, BP neural network based image segmentation technique for small sized league soccer robot team system, Journal of Mechanical \& Electrical Engineering, 28(1), 2011, 79-82.

[3]. K. Qin, K. Xu, F.L. Liu and D.Y. Li, Image segmentation based on histogram analysis utilizing the cloud model, Computers \& Mathematics with Applications, 62(7), 2011, 2824-2833.

[4]. S. Liu, Image segmentation technology of the Ostu method for image materials based on binary PSO algorithm, Advances in Intelligent and Soft Computing, 104,2011, 415-419.

[5]. X.J. Lei and A.L. Fu, 2-D maximum-entropy thresholding image segmentation method based on second-order oscillating PSO, Proc. 5th International Conference on Natural Computation, Tianjin, China, 2009, 161-165.

[6]. J.Z. Fei, Research and design of SSL vision system based on FPGA, master's diss., Hubei University of Technology, Wuhang, China, 2011.

[7]. C. Dagli, and T.S. Huang, A framework for grid-based image retrieval, Proc. 17th International Conference on Pattern Recognition, Cambridge, UK. 2004, 1021-1024.

[8]. X.Q. Deng, The research on the robot-soccer visual subsystem, master’s diss., Xihua University, Chengdu, China, 2007. 